\title{
Occurrence of Discussion about Lung Cancer Screening Between Patients and Healthcare Providers in the USA, 2017
}

\author{
Samir Soneji ${ }^{1}$ JaeWon Yang ${ }^{2}$ - Nichole T. Tanner ${ }^{3,4} \cdot$ Gerard A. Silvestri ${ }^{3}$
}

Published online: 9 March 2019

\begin{abstract}
Computed tomography lung cancer screening reduces lung cancer mortality. However, screening is underutilized. This study assesses the extent to which providers discuss lung cancer screening with their patients, as a lack of discussion and counseling may serve as a potential cause of low utilization rates. Data from 1667 adults aged 55-80 years sampled in the 2017 Health Information National Trends Survey was utilized. A weighted multivariable logistic regression model was fit with past-year discussion about lung cancer screening with a provider as the outcome. The adjusted odds of discussion were higher for current cigarette smokers compared to non-cigarette smokers (adjusted odds ratio $=3.91 ; 95 \%$ confidence interval $[\mathrm{CI}], 1.75$ to 8.74 ). Despite higher odds, the absolute prevalence was low with only 18\% (95\% CI, 11.8 to $24.2 \%$ ) of current adult smokers reporting a past-year discussion. Knowledge of screening from trusted sources of medical information, such as doctors, can increase screening rates and may ultimately reduce lung cancer mortality.
\end{abstract}

Keywords Lung cancer $\cdot$ Screening $\cdot$ Doctor-patient

\section{Introduction}

Utilization of low-dose computed tomography (CT) lung cancer screening (LCS) remains low despite demonstrated efficacy, clinical recommendation, and insurance coverage [1,2]. In 2015 , only $3.9 \%$ of screening-eligible persons reported CT LCS within the past year [3]. Screening rates may be low for several reasons. First, patients may not seek screening due to

Electronic supplementary material The online version of this article (https://doi.org/10.1007/s13187-019-01510-9) contains supplementary material, which is available to authorized users.

\footnotetext{
Samir Soneji

samir.soneji@dartmouth.edu
}

1 Norris Cotton Cancer Center, Geisel School of Medicine at Dartmouth, Dartmouth College, Dartmouth Institute for Health Policy \& Clinical Practice, Hanover, One Medical Center Drive, Lebanon, NH 03756, USA

2 Warren Alpert Medical School, Brown University, Providence, RI, USA

3 Thoracic Oncology Research Group and Division of Pulmonary Critical Care Medicine, Medical University of South Carolina, Chapel Hill, NC, USA

4 Ralph H. Johnson Veterans Affairs Hospital and Health Equity and Rural Outreach Innovation Center, Charleston, SC, USA lack of knowledge. Second, healthcare providers may lack a detailed understanding of the potential benefits and harms of CT LCS and, therefore, fail to initiate conversations with their patients. We undertook this study to estimate the prevalence of these discussions about LCS between patients and their providers.

\section{Methods}

\section{Data}

This study utilized data from the Health Information National Trends Survey (HINTS), a nationally representative, crosssectional survey administered by the US National Cancer Institute to evaluate the use of cancer-related information. HINTS 5, Cycle 1 data $(N=3285)$ were collected from January-May 2017 and utilized a two-stage sampling design. This study focused on 1667 respondents whose age fell within the USPSTF recommended age interval for LCS (5580 years).

\section{Outcome and Covariates}

The outcome was past-year discussion with a healthcare provider about LCS ("At any time in the past year, have 
you talked with your doctor or other health professional about having a test to check for lung cancer?"). Sociodemographic characteristics included age, race/ethnicity, and education. Health-related characteristics included self-reported health, history of cancer, certain comorbidities (diabetes, hypertension, heart condition, and chronic lung disease), regular source of care, and health insurance status. Smoking status included never, former, and current cigarette smokers. Level of trust in information about health or medical topics was categorized as a lot, some, a little, or none and was assessed from various sources, including the government, family, and a doctor or other healthcare professional (hereafter, "provider"). See Supplementary Table 1 for details.

\section{Analysis}

First, the level of trust about health information from providers was compared to the corresponding level of trust from other sources. Second, the prevalence of discussions with providers about LCS within the past year was estimated by smoking status. Third, a weighted multivariable logistic regression model was fit with past-year LCS discussion as the outcome; covariates included socio-demographic and health characteristics, smoking status, and level of trust in information from a provider.

\section{Results}

\section{Study Population}

The percentage of the population aged 55-59, 60-64, 65-69, and $70-80$ years were $27.4 \%, 24.5 \%, 20.5 \%$, and $27.6 \%$, respectively. The population was $48.1 \%$ male; $76.4 \%$ nonHispanic white, $8.6 \%$ non-Hispanic black, and 9.2\% Hispanic; and $96.1 \%$ had health insurance (Table 1). The percentage of never, former, and current cigarette smokers were $52.4 \%, 32.8 \%$, and $14.8 \%$, respectively. Most respondents reported their health as either very good $(33.8 \%)$ or good (35.8\%). Hypertension was commonly reported (58.1\%), whereas chronic lung disease and a heart condition were less frequently reported (15.5\% and $12.9 \%$, respectively).

\section{Trust Information about Health or Medical Topics}

Providers were the most trusted source of information (Supplementary Fig. 1). For example, 70.5\% of adults reported that they would trust information about health or medical topics from a provider "a lot" compared to only $21.8 \%$ of adults reporting this same level of trust if the source of information was the government.
Table 1 Characteristics of study population

\begin{tabular}{|c|c|c|c|}
\hline & $N$ & Wgt. Prev. & $95 \% \mathrm{CI}$ \\
\hline \multicolumn{4}{|l|}{ Age group (years) } \\
\hline $55-59$ & 374 & $27.4 \%$ & $(24.3 \%, 30.6 \%)$ \\
\hline $60-64$ & 404 & $24.5 \%$ & $(21.6 \%, 27.4 \%)$ \\
\hline $65-69$ & 412 & $20.5 \%$ & $(18.0 \%, 22.9 \%)$ \\
\hline $70-80$ & 477 & $27.6 \%$ & $(24.7 \%, 30.5 \%)$ \\
\hline \multicolumn{4}{|l|}{ Sex } \\
\hline Male & 705 & $48.1 \%$ & $(44.8 \%, 51.5 \%)$ \\
\hline Female & 938 & $51.9 \%$ & $(48.5 \%, 55.2 \%)$ \\
\hline \multicolumn{4}{|l|}{ Race/ethnicity } \\
\hline Non-Hispanic White & 1046 & $76.4 \%$ & $(73.6 \%, 79.2 \%)$ \\
\hline Non-Hispanic Black & 196 & $8.6 \%$ & $(6.8 \%, 10.4 \%)$ \\
\hline Hispanic & 160 & $9.2 \%$ & $(7.3 \%, 11.2 \%)$ \\
\hline Non-Hispanic Asian & 54 & $3.1 \%$ & $(2.0 \%, 4.2 \%)$ \\
\hline Non-Hispanic Other & 54 & $2.8 \%$ & $(1.7 \%, 3.8 \%)$ \\
\hline \multicolumn{4}{|l|}{ Educational attainment } \\
\hline Less than high school & 121 & $8.9 \%$ & $(6.6 \%, 11.2 \%)$ \\
\hline High school graduate & 373 & $27.6 \%$ & $(24.6 \%, 30.5 \%)$ \\
\hline At least some college & 1161 & $63.5 \%$ & $(60.2 \%, 66.8 \%)$ \\
\hline Health insurance coverage & 1591 & $96.1 \%$ & $(94.7 \%, 97.5 \%)$ \\
\hline \multicolumn{4}{|l|}{ Cigarette smoking status } \\
\hline Never & 907 & $52.4 \%$ & $(49.1 \%, 55.8 \%)$ \\
\hline Former & 528 & $32.8 \%$ & $(29.7 \%, 35.9 \%)$ \\
\hline Current & 224 & $14.8 \%$ & $(12.1 \%, 17.4 \%)$ \\
\hline Regular healthcare provider & 1647 & $78.6 \%$ & $(75.8 \%, 81.5 \%)$ \\
\hline \multicolumn{4}{|l|}{ Self-reported health } \\
\hline Excellent & 160 & $8.9 \%$ & $(7.1 \%, 10.6 \%)$ \\
\hline Very good & 559 & $33.9 \%$ & $(30.8 \%, 37.1 \%)$ \\
\hline Good & 594 & $35.8 \%$ & $(32.6 \%, 38.9 \%)$ \\
\hline Fair & 291 & $18.1 \%$ & $(15.4 \%, 20.7 \%)$ \\
\hline Poor & 49 & $3.4 \%$ & $(2.0 \%, 4.7 \%)$ \\
\hline Ever had cancer & 1664 & $16.9 \%$ & $(14.7 \%, 19.0 \%)$ \\
\hline Diabetes & 1639 & $15.5 \%$ & $(12.8 \%, 18.2 \%)$ \\
\hline Heart condition & 1643 & $12.9 \%$ & $(10.7 \%, 15.2 \%)$ \\
\hline Chronic lung disease & 1635 & $58.1 \%$ & $(54.8 \%, 61.4 \%)$ \\
\hline \multicolumn{4}{|l|}{ Body mass index } \\
\hline Underweight & 16 & $0.7 \%$ & $(0.3 \%, 1.1 \%)$ \\
\hline Normal & 423 & $26.1 \%$ & $(23.1 \%, 29.0 \%)$ \\
\hline Overweight & 583 & $35.3 \%$ & $(32.1 \%, 38.5 \%)$ \\
\hline Obese & 602 & $37.9 \%$ & $(34.6 \%, 41.2 \%)$ \\
\hline
\end{tabular}

$N$, count; Wgt. Prev., weighted prevalence; $C I$, confidence interval

\section{Lung Cancer Screening by Cigarette Smoking Status}

Eighteen percent of current cigarette smokers reported a discussion with their provider about LCS within the past year $(95 \%$ CI, 11.8 to $24.2 \%$; Fig. 1). Discussion prevalence was lower for former cigarette smokers $(10.5 \%$; $95 \% \mathrm{CI}, 7.3$ to $13.8 \%)$ and never cigarette smokers $(3.8 \%$; $95 \% \mathrm{CI}, 2.1 \%$ to $5.6 \%)$. 
Fig. 1 Prevalence of discussion with doctor or other healthcare professional about lung cancer screening within past year by cigarette smoking status. Source: Authors' analysis of 2017 Health Information National Trends Survey 5, Cycle 1
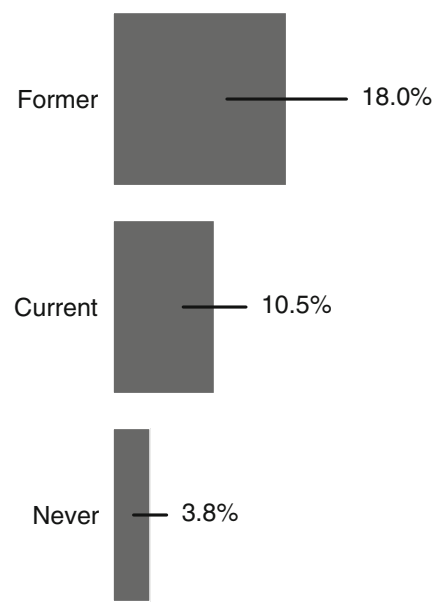

\begin{tabular}{|c|c|c|c|c|c|c|c|c|c|}
\hline 0 & 10 & 20 & 30 & 40 & 50 & 60 & 70 & 80 & 90 \\
\hline
\end{tabular}

\section{Correlates of Lung Cancer Screening Discussion}

The odds of discussing LCS with a provider within the past year were higher for current (adjusted odds ratio $[\mathrm{aOR}]=3.91$; $95 \% \mathrm{CI}, 1.75$ to 8.74$)$ and former $(\mathrm{aOR}=2.06 ; 95 \% \mathrm{CI}, 1.10$ to 3.86) cigarette smokers compared to never cigarette smokers (Table 2). Odds of discussion were also higher for adults aged 65-69 years compared to adults aged 55-59 years $(\mathrm{aOR}=2.81 ; 95 \% \mathrm{CI}, 1.24$ to 6.36$)$, those who ever had cancer $(\mathrm{aOR}=1.99 ; 95 \% \mathrm{CI}, 1.14$ to 3.47$)$, and those with chronic lung disease ( $\mathrm{aOR}=3.0295 \% \mathrm{CI}, 1.63$ to 5.61). Odds of discussion were not significantly higher for adults with health insurance compared to those without $(\mathrm{aOR}=1.48 ; 95 \% \mathrm{CI}$, 0.28 to 7.95 ).

\section{Discussion}

Our study suggests the low prevalence of discussions about LCS between patients and providers may partly explain the low prevalence of CT LCS. Several barriers may prevent these discussions, including confusion about eligibility criteria, lack of sufficient time during a routine office visit, and discomfort with managing screen-detected findings [4]. Even when conversations do occur, they may be poor in quality [5]. A recent qualitative analysis of conversations between providers and patients about LCS concluded these discussions frequently scored low on a validated scale of shared decision making, rarely discussed potential harms, and did not utilize patient education materials, despite evidence that they help patients reach an informed decision [6, 7]. More rigorous training for providers in shared decision-making - a Centers for Medicare and Medicaid Services requirement of LCS - could improve discussion quality [8].
Table 2 Weighted multivariable logistic regression results: discussion with doctor or other healthcare professional about lung cancer screening within past year

\begin{tabular}{ll}
\hline Covariate & Adj. OR $(95 \%$ CI) \\
\hline Age group (ref: 55-59 years) & \\
$60-64$ & $1.51(0.62,3.67)$ \\
$65-69$ & $2.81(1.24,6.36)$ \\
$70-80$ & $1.43(0.62,3.29)$ \\
Female (ref: male) & $0.58(0.32,1.05)$ \\
Race/ethnicity (Ref: Non-Hispanic White) & \\
Non-Hispanic Black & $1.45(0.58,3.60)$ \\
Hispanic & $1.01(0.36,2.84)$ \\
Non-Hispanic Asian & $2.50(0.70,8.91)$ \\
Non-Hispanic Other & $2.45(0.70,8.51)$ \\
Educational attainment (ref: < high school) & \\
High school graduate & $0.38(0.09,1.57)$ \\
At least some college & $0.34(0.09,1.33)$ \\
Health insurance (ref: no) & $1.48(0.28,7.95)$ \\
Smoking status (ref: never) & \\
Current & $3.91(1.75,8.74)$ \\
Former & $2.06(1.10,3.86)$ \\
Trust information from a doctor ${ }^{1}$ (ref: not at all) & \\
A lot & $0.55(0.04,7.95)$ \\
Some & $0.92(0.06,13.66)$ \\
A little & $0.32(0.02,6.35)$ \\
Regular healthcare provider (ref: no) & $1.48(0.64,3.41)$ \\
Self-reported health (ref: good) & \\
Excellent & $0.38(0.10,1.44)$ \\
Very good & $1.03(0.56,1.90)$ \\
Fair & $0.59(0.28,1.24)$ \\
Poor & $1.03(0.29,3.68)$ \\
Ever had cancer (ref: no) & $1.99(1.14,3.47)$ \\
Diabetes & $1.23(0.65,2.31)$ \\
Heart condition & $1.09(0.52,2.31)$ \\
Hypertension & $0.94(0.51,1.72)$ \\
Chronic lung disease & $3.02(1.63,5.61)$ \\
Body mass index (ref: normal) & \\
Underweight & $1.57(0.21,11.64)$ \\
Overweight & $0.82(0.41,1.65)$ \\
Obese & $0.82(0.39,1.73)$ \\
&
\end{tabular}

Adj. OR, adjusted odds ratio; $C I$, confidence interval; Ref, reference

${ }^{1}$ Trust information about health or medical topics from a doctor or other healthcare professional 
Better patient engagement could increase the frequency and quality of discussions. Patients interested in LCS may not fully comprehend existing educational materials because they are written at exceedingly high readability levels [9]. Professional medical societies could address this current gap by producing educational materials written at an appropriate reading level.

We note several limitations. First, the data collected in HINTS did not allow us to estimate pack-year smoking history and, therefore, screening eligibility under USPSTF recommendations. However, in $2017,41.6 \%$ of current smokers aged 55-80 years were $\geq 30$ pack-year smokers and another 19.9\% were 20-29 pack-year smokers (Supplementary Fig. 2). Current smokers with 20-29 pack-year smoking histories - included in our analysis - may also benefit from CT LCS [10]. Second, HINTS did not specify the modality of screening. Some physicians may have discussed chest Xray screening even though it is not clinically recommended. Thus, our study may conservatively estimate the prevalence of discussion between physicians and patients on CT LCS.

An important precursor to patients receiving routine $\mathrm{CT}$ LCS is knowledge and awareness. Providers often serve as trusted sources of information, and discussions that appropriately explain potential risks and benefits can help increase awareness and deliver patient-centered care. If these discussions encourage high-risk patients to screen routinely, the burden of lung cancer mortality may decrease.

Publisher's Note Springer Nature remains neutral with regard to jurisdictional claims in published maps and institutional affiliations.

\section{References}

1. National Lung Screening Trial Research Team, Aberle DR, Adams $\mathrm{AM}$, et al. Reduced lung-cancer mortality with low-dose computed tomographic screening. N Engl J Med 2011;365(5):395-409. doi: https://doi.org/10.1056/NEJMoa1102873

2. Soneji S, Yang J, Tanner NT, Dang R, Silvestri GA, Black W (2017) Underuse of chest radiography versus computed tomography for lung cancer screening. Am J Public Health 107(8):12481250. https://doi.org/10.2105/AJPH.2017.303919

3. Jemal A, Fedewa SA (February 2017) Lung cancer screening with low-dose computed tomography in the United States-2010 to 2015. JAMA Oncol 3:1278-1281. https://doi.org/10.1001/ jamaoncol.2016.6416

4. Kanodra NM, Pope C, Halbert CH, Silvestri GA, Rice LJ, Tanner NT (2016) Primary care provider and patient perspectives on lung cancer screening. A qualitative study. Ann Am Thorac Soc 13(11): 1977-1982. https://doi.org/10.1513/AnnalsATS.201604-286OC

5. Wiener RS, Koppelman E, Bolton R, Lasser KE, Borrelli B, Au DH, Slatore CG, Clark JA, Kathuria H (2018) Patient and clinician perspectives on shared decision-making in early adopting lung cancer screening programs: a qualitative study. J Gen Intern Med 33(7): 1035-1042. https://doi.org/10.1007/s11606-018-4350-9

6. Stacey D, Bennett CL, Barry MJ et al (2011) Decision aids for people facing health treatment or screening decisions. Cochrane Database Syst Rev (10):CD001431. https://doi.org/10.1002/ 14651858.CD001431.pub3

7. Stacey D, Légaré F, Lewis KB (2017) Patient decision aids to engage adults in treatment or screening decisions. JAMA 318(7):657658. https://doi.org/10.1001/jama.2017.10289

8. Bieber C, Nicolai J, Hartmann M, Blumenstiel K, Ringel N, Schneider A, Härter M, Eich W, Loh A (2009) Training physicians in shared decision-making-who can be reached and what is achieved? Patient Educ Couns 77(1):48-54. https://doi.org/10. 1016/j.pec.2009.03.019

9. Hansberry DR, White MD, D’Angelo M et al (2018) Lung cancer screening guidelines: how readable are internet-based patient education resources? AJR Am J Roentgenol 211(1):W42-W46. https:// doi.org/10.2214/AJR.17.19042

10. Pinsky PF, Zhu CS, Kramer BS. Lung cancer risk by years since quitting in 30+ pack year smokers. J Med Screen 2015;22(3):151157. doi:https://doi.org/10.1177/0969141315579119 